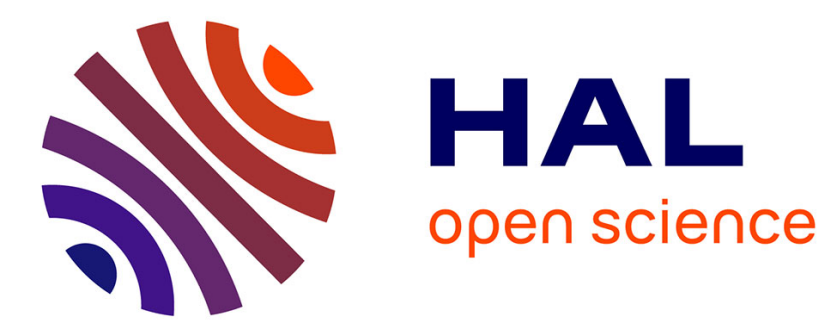

\title{
Qu'en est-il quatre ans après la tornade de 1982 dans le Massif central?
}

\author{
Thierry Thuret
}

\section{To cite this version:}

Thierry Thuret. Qu'en est-il quatre ans après la tornade de 1982 dans le Massif central?. Revue forestière française, 1987, 39 (4), pp.378-386. 10.4267/2042/25809 . hal-03424890

\section{HAL Id: hal-03424890 \\ https://hal.science/hal-03424890}

Submitted on 10 Nov 2021

HAL is a multi-disciplinary open access archive for the deposit and dissemination of scientific research documents, whether they are published or not. The documents may come from teaching and research institutions in France or abroad, or from public or private research centers.
L'archive ouverte pluridisciplinaire HAL, est destinée au dépôt et à la diffusion de documents scientifiques de niveau recherche, publiés ou non, émanant des établissements d'enseignement et de recherche français ou étrangers, des laboratoires publics ou privés. 


\title{
QU'EN EST-IL QUATRE ANS APRES LA TORNADE DE 1982 DANS LE MASSIF CENTRAL ?
}

\author{
T. THURET
}

Que faut-il penser des remarques du style "l'aide aux chablis a été un "plan Marshall pour la forêt" ou "les chablis ont été pour la forêt ce qu'un incendie est pour une scierie"?

Si quatre ans sont peu de chose pour constater des résultats sur le plan forestier, c'est cependant, sur le plan industriel, un délai suffisant pour tirer les premiers enseignements d'une action menée, rappelons-le, à ce niveau industriel, pour catalyser des réactions en amont de la filière.

Les bilans de la mobilisation des chablis et des équipements consentis pour leur transformation venant d'être faits, notre propos sera plutôt de faire un point sur la santé économique et l'état d'esprit des partenaires de la filière-bois à la suite de la catastrophe qui a frappé le Massif Central en novembre 1982 .

Sous le coup de l'émotion et devant l'étendue du désastre et les difficultés de chiffrer leur ampleur, les dégâts et les moyens à mettre en œuvre pour les réparer avaient été largement surévalués. La confrontation des réalisations et des crédits demandés à l'époque nécessite, quatre ans plus tard, des justifications sinon des explications aux socio-professionnels - et aux élus quand les collectivités ont participé à l'action.

\section{RÉALITÉ DES DÉGÂTS}

L'écart entre les surfaces reboisées ou à reboiser en 1987 et les surfaces dont la reconstitution avait été envisagée est de près de la moitié ( 12000 ha sur les 23000 ha annoncés pour l'Auvergne, et 1150 ha sur les 2800 ha annoncés pour le Limousin). II a fallu expliquer que certaines surfaces n'étaient que partiellement sinistrées, ne justifiant pas une reconstitution et que la réalité des dégâts était à $75 \%$ des estimations mais aussi que sur ces $75 \%$, un bon tiers ne serait jamais exploité, nettoyé et replanté...

II faut bien distinguer en effet l'évaluation des volumes - très précise, notamment en forêt soumise - et celle des surfaces, plus difficile, faute de repères et d'accès. La multitude de 
"mini-trouées", la décision de ne pas exploiter à blanc certaines parcelles en y laissant des bouquets d'arbres - d'ailleurs difficiles à vendre à l'époque - et le démarrage de régénérations sont de nature à expliquer ces écarts.

\section{LA MOBILISATION}

Les bilans successifs sont éloquents: "les meubles ont été sauvés", puisque $70 \%$ du volume des chablis, essentiellement les gros diamètres, étaient exploités 7 mois après la tornade. L'exploitation des bois d'industrie - notamment en Limousin et en Combrailles où ils constituaient une grande partie du volume des jeunes peuplements sinistrés - a duré par contre jusqu'au printemps 1985, époque où les lots étaient finalement refusés par tous les utilisateurs européens...

On pouvait donc, 18 mois après la tempête, considérer que l'essentiel de la mobilisation était terminé et que les bois restants (les petits chablis disséminés, isolés ou non rentables à exploiter) ne seraient jamais commercialisés. Quatre ans après, il y a effectivement de nombreuses perches penchées et renversées dans les pineraies, des Sapins cassés, des Chênes ébranchés, sans parler des arbres affaiblis qui n'ont pas été exploités, ont été parasités par les bostryches et présentent aujourd'hui le triste spectacle de leurs fûts pelés...

Si les mesures prises au niveau de l'exploitation et de la première transformation sont effectivement la cause de cette mobilisation exemplaire et ont permis aux industries locales de stocker $15 \%$ et scier $25 \%$ de ces chablis, il faut quand même signaler l'importante décote des bois résineux - les feuillus se sont mieux tenus - et l'effondrement des prix pour les petits bois, ruinant pour 30 ans maints propriétaires et collectivités...

II faut surtout noter, comme retombées de l'effort consenti par les pouvoirs publics, les points positifs ou négatifs suivants.

\section{Équipement des entreprises}

Grâce au système très souple des aides (près de 800 dossiers furent traités), de nombreuses entreprises ont modernisé leur matériel el de nombreux agriculteurs sont équipés en arceaux de sécurité, treuils et autres fendeuses de bûches. Cet accroissement de potentiel favorise aujourd'hui l'exploitation des bois bostrychés et des " produits accidentels " encore disséminés sur les surfaces à replanter. Si on peut regretter l'absence de matériels français - bien que certains équipements scandinaves adaptés à l'exploitation par coupe rase de vastes surfaces et utilisés en 1984 dans les jeunes pineraies des Combrailles soient impossibles à rentabiliser en exploitation normale - et déplorer que de nombreux bûcherons étrangers cherchent encore localement du travail, on doit considérer comme positif le développement de petites entreprises (2 à 3 personnes) dont le potentiel a été multiplié. Sont également des conséquences positives : la fixation en Auvergne d'un entrepreneur norvégien équipé d'une tête d'ébranchage, l'équipement de la région Limousin, avec l'aide du Conseil régional, de 4 ébrancheurs-tronçonneurs type SIFER, et la multiplication de vocations pour le travail en forêt susceptibles de rattraper le retard important pris par le Massif Central en matière d'éclaircies...

\section{Équipement des massifs}

La desserte était soit adaptée au traitement en futaie jardinée dans les zones de moyenne montagne et ne permettait pas la sortie de volumes aussi inhabituels, soit réellement absente dans certaines zones de petites propriétés anciennement reboisées. 
450 kilomètres de pistes ont donc dũ être repris ou ouverts ( $175 \mathrm{~km}$ ouverts en Auvergne) pour évacuer les bois de ces zones pas ou insuffisamment desservies, ce qui a évidemment facilité la reconstitution et permettra, à l'avenir, une sylviculture plus dynamique (éclaircies plus rentables par exemple).

La retombée la plus importante est que l'équipement des massifs s'est imposé aux élus et aux propriétaires comme la priorité majeure de leur action en amont de la filière-bois. En Limousin, le Conseil régional finance les schémas directeurs d'équipement forestier et cofinance la réalisation des dessertes; une action exemplaire de création "d'associations syndicales de desserte forestière ", démarrée par nos services du Cantal et relayée, début 1986, dans toute l'Auvergne par le Centre régional de la Propriété forestière avec des financements de la Région et du Fonds forestier national, fait " tache d'huile" et constitue une des réponses aux questions d'approvisionnement des industries locales du bois.

\section{Organisation des transports}

Malgré les efforts de la SNCF - qui a même remis en service la pittoresque voie " du Puy à Saint-Alyre d'Arlanc " - le transport de telles quantités de bois $(800000 \mathrm{t})$ par fer a été handicapé par le sous-équipement des gares en matière de manutention du bois.

Mais c'est la suppression, au $1^{\text {er }}$ janvier 1984, des avantages tarifaires dont le bois bénéficiait auprès de la SNCF (annexe B ter) qui a permis de remédier à certains de ces handicaps. Des aides publiques compensatoires à hauteur de 5,78 millions de francs ont généré pour 20,5 millions de francs d'investissements dans huit gares SNCF, deux centres de regroupement de bois pour l'usine de pâte à papier de Tarascon et pour l'achat de matériels de transport et de manutention notamment en Auvergne, Limousin et Languedoc-Roussillon. Les professionnels limousins et la SNCF ont en outre développé la formule "TARC " (trains à rames convergentes) et optimisé les coûts de transport, ce qui permet, quatre ans après, de faire face à l'accroissement des volumes destinés à l'usine CDRA de Tarascon.

Les “points noirs " routiers (Lozère) et l'insuffisance du réseau routier secondaire lors du dégel sont également clairement apparus après cette tempête.

S'il est encore trop tôt pour juger de l'impact de ces investissements, les contacts pris avec les autres administrations à cette occasion les ont sensibilisées à nos difficultés et la filière-bois était considérée comme un volet prioritaire dans l'étude des transports menée fin 1986 en Auvergne.

\section{$\stackrel{* *}{* *}$}

II est notoire que les grands chablis européens donnent un supplément de travail à de nombreux bûcherons, débardeurs, transporteurs et scieurs, tant locaux qu'extérieurs et même étrangers. Au-delà des problèmes d'organisation, des accidents, parfois mortels, des bonnes affaires (ou des mauvaises), des habituels profiteurs et des éternelles victimes qui ont marqué cette énorme "coupe de $10000000 \mathrm{~m}^{3}$ ", il y a la prise de conscience locale des problèmes de l'amont de la filière-bois. Elle se traduit, quatre ans après, par une réelle prise en compte des problèmes de sécurité, de formation professionnelle (multiplication des formations et des perfectionnements des ouvriers), d'organisation de l'offre et des problèmes d'approvisionnement ultérieur des entreprises (création en 1985 de la bourse auvergnate des travaux forestiers, sur le modèle de la bourse limousine de 1981 qui a joué un rôle important dans la mobilisation des chablis de cette région). Elle a certainement favorisé la menée à bien des "volets forestiers" des contrats de plan locaux ( $18 \%$ des crédits régionaux consacrés aux activités "rurales " en Limousin, $25 \%$ en Auvergne alors que la moyenne nationale est de $7,6 \%$ ) et le développement qu'elle a induit permettra, nous l'espérons, une certaine pérennité des efforts des élus pour leurs forêts. 


\section{LA RECONSTITUTION}

La nécessité de nettoyer, de préparer et de planter dans les meilleurs délais de telles surfaces, avait conduit les pouvoirs publics à privilégier les chantiers importants quitte, pour le Conseil régional d'Auvergne, faute d'utilisateurs des crédits obtenus, à instituer, quatre ans après, des bons-subventions pour le reboisement de parcelles de 50 ares !...

La simple exploitation, courant 1983, des surfaces sinistrées, remettait à 1984 la plupart des grands chantiers mais, alors que beaucoup de travaux étaient prêts à lancer, les premiers grands reboisements ne démarrèrent qu'à l'automne 1985.

Faut-il le déplorer alors que les grands gels puis la longue sécheresse (mars-décembre) de 1985 puis de nouveau un rude hiver et une nouvelle sécheresse en 1986 auraient sans doute mis à mal la majorité des reboisements de plaine et de piedmont et que ce retard a permis d'éviter à l'Hylobe de multiplier ses dégâts sur des millions de jeunes plants ?

Quatre ans après, en pleine campagne de reconstitution, quels enseignements - autres que la longueur du délai nécessaire à la mise en œuvre des reboisements - tirer de ce premier volet?

\section{Sur les procédures}

La mise en place de crédits exceptionnels est presque aussi longue que le temps nécessaire pour convaincre les propriétaires, ruinés parfois, de réinvestir en forêt, monter les dossiers, lancer les appels d'offres, etc... Mais on peut éviter de retarder les premières plantations en mettant plus vite les crédits en place: les collectivités, par exemple, n'ont jamais voulu lancer les travaux avant d'avoir les notifications de crédits... d'où salissement des terrains et coûts supplémentaires...

\section{Sur les techniques}

La taille des chantiers a permis l'emploi de techniques et de matériels de préparation des sols rarement utilisés dans notre zone de moyenne montagne et de petites propriétés: lame Rome et billonneur en forêt de Tronçais, par exemple... La fragilité des sols volcaniques a permis d'apprécier le rateau Fleco et surtout la lame à dents escamotables; certaines fortes pentes ont nécessité l'emploi d'un grappin andaineur à vérins de positionnement, évidemment surnommè "Dahu "... Rien de nouveau quant aux techniques de plantation mais, quant aux choix des essences, nous avons noté la grande faveur dont a joui le Douglas, essence à croissance plus rapide que l'Épicéa et ayant mieux résisté à la tornade - faveur qu'il a fallu tempérer chez certains propriétaires de terrains à plus de 1000 mètres d'altitude ou dans des zones tout à fait impropres. Cette faveur a d'ailleurs nécessité, des services de terrain, le refus de plants commandés sans discernement par des reboiseurs, du fait de la pénurie locale.

Notons entin que les densités de plantations sont devenues proches des minima que prèconisent les responsables du FFN et que des essais de plantations à grands espacements ou d'essences à risques ont été tentés lorsque les propriétaires, faute de pouvoir prétendre aux aides publiques, étaient libres de faire de tels essais.

\section{Sur les coûts}

Ne pouvant les comparer que sur deux exercices très inégaux - où on constate leur stabilité, sinon certaines baisses - il ne serait pas sérieux d'avancer que la présence des leaders nationaux du reboisement entraîne la disparition ou la conversion de petites entreprises locales, même si certaines se sont déjà lancées dans le débardage, plus rémunérateur que la préparation du terrain au rateau Fleco... 


\section{T. THURET}

II faut signaler une sorte d'osmose entre le Limousin - richement doté d'entreprises de reboisement, cantonnées à 2000 ha/an alors qu'il s'y plantait jusqu'à 7000 ha - et l'Auvergne, sous-équipée, où elles continueront à œuvrer quelques années pour effectuer les dégagements nécessaires.

$$
*^{* * *}
$$

Cette œuvre de reconstitution a montré l'intérêt, sinon la nécessité, faute de main-d'œuvre, de disposer de gros matériels et incité plusieurs entreprises à s'équiper et plusieurs jeunes à choisir, là aussi, des formations professionnelles plus adaptées aux réalités forestières de demain.

\section{LA TRANSFORMATION}

Le souci d'accroître la capacité des scieries locales en assurant le stockage d'une partie des bois exploités et en développant leur potentiel de transformation avait guidé, en priorité, l'action des pouvoirs publics. Cela n'aurait, bien sûr, pas suffi à mobiliser un tel volume sans le recours à des entreprises extérieures, françaises ou étrangères, incitées à s'approvisionner grâce aux aides au transport dont le bilan vient d'être fait par Ch. Barthod.

Quelle est, aujourd'hui, la situation dans les entreprises?

\section{Le stockage}

Contrairement au Limousin où les scieurs n'ont stocké que dans la mesure de leurs possibilités, l'ampleur du stockage par les entreprises auvergnates et par conséquent la sous-consommation des aides au transport prévues pour les entreprises extérieures, ont surpris la plupart des responsables. Ils pensent, qu'á l'époque, les prix des sciages étaient à la hausse et que les industriels locaux ont pris le risque de se "charger" (170 millions de francs de prêts bonifiés) pour ne pas avoir à chercher trop loin leur approvisionnement au cours des années suivantes.

Mais la tendance s'étant inversée, le coût de ce stockage et la raréfaction des gros bois se faisant sentir, les échéances des prêts s'approchant, les scieurs ont dû "déstocker" dès le début 1985.

Il a même fallu, au courant de cette année, de nombreuses réunions avec les organismes financiers pour réaménager les échéanciers des scieurs ayant les plus grosses difficultés: investissements importants, poids des stocks de coffrage, invendable en tant que tel, problème général des taux des emprunts en hausse (7\% puis $9 \%$ ) et de l'inflation en baisse...

Tout est à peu près rentré dans l'ordre - sans doute au prix d'une certaine fragilitè financière des entreprises - et la qualité des sciages issus des bois stockés a rassure la plupart des scieurs et de leurs clients. On peut estimer que $80 \%$ des grumes stockées ont déjà été sciées, faute de quantités suffisantes de bois de grosseurs ou qualités équivalentes et surtout parce que de lourdes échéances le nécessitaient, ou vendues en l'état, notamment les Chênes de l'Allier.

Mais si le stock des grumes de 1982 est quasiment épuisé, les installations d'aspersion ne sont pas encore amorties et certains scieurs ont décidé de tirer parti de cette technique donnant à leurs sciages un aspect "bois du Nord" qui peut s'avérer intèressante sur le plan commercial. 


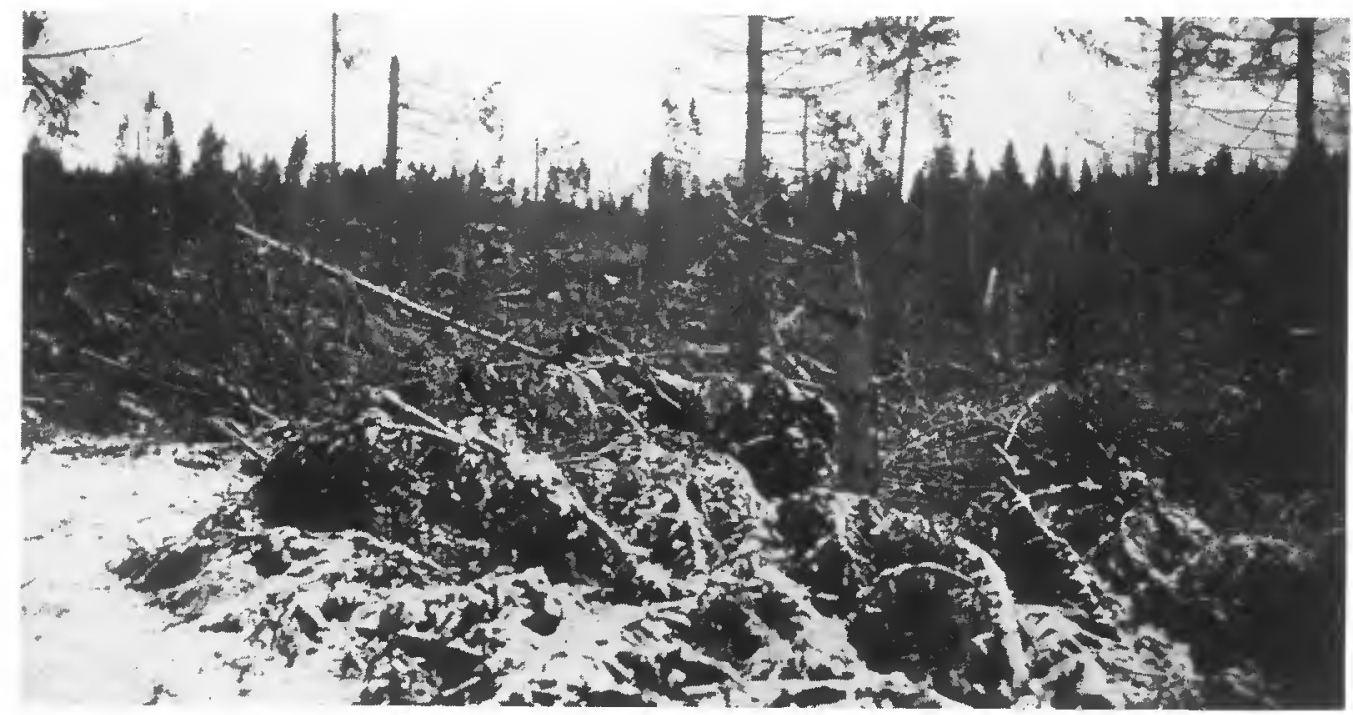

Photo S.R.F.B.

\section{L'équipement des entreprises}

A la suite de la tempête de 1982, le Fonds forestier national a attribué des prêts (environ 28 millions de francs) à une trentaine de scieurs du Massif Central pour moderniser leurs installations: une enquête, en cours, de la Direction des Forêts, permettra de mesurer l'impact de ces prêts sur la capacité et la structure des entreprises.

Si nous savons déjà que l'augmentation théorique de la capacité de production est de $60000 \mathrm{~m}^{3} / \mathrm{an}$, il est difficile, dans l'augmentation de $100000 \mathrm{~m}^{3}$ des sciages produits entre 1982 et 1985 , de faire la part entre la capacité ancienne sous-exploitée avant 1982 et l'utilisation, en totalité, de la capacité nouvelle. L'essentiel reste que ces investissements ont permis de développer la présence des industriels du Massif Central sur les marchés nationaux et même à l'exportation, et par là de les sensibiliser aux difficultés, aux exigences qualitatives et surtout aux nécessités de suivi et de service qui constituent la "gestion de la qualité "à laquelle les interprofessions du bois sont aujourd'hui confrontées.

Des efforts d'investissements ont été consentis dès 1984 pour faire face à ces exigences de qualité (tri, classement, séchage, sciage d'équerre, colisage, etc...), et la simplicité de mise en cuvre des aides du Fonds interministériel pour le Développement de l'Aménagement rural (FIDAR) aux entreprises de la filière-bois y a beaucoup contribué.

D'autres aides publiques, adaptées aux différents maillons de la filière, ainsi que les primes régionales ont été, sinon découvertes, en tous cas mieux utilisées puisque leur attribution était souvent conditionnée, au terme d'analyses financières et même de vérifications, par une restructuration financière des entreprises.

$$
\text { *** }
$$

La tornade de 1982, qui avait imposé la réunion des partenaires de la filière sur un objectif économique et un pari à tenir - et non sur les habituelles considérations socio-professionnelles - a sans doute contribué à l'instauration d'un état d'esprit que le vaste "brain storming "des contrats de plan et chartes de modernisation des scieries a encore développé. 


\section{T. THURET}

La mise en place d'interprofessions et l'animation de la filière qu'elles permettent ont “ouvert les yeux " de nombreux scieurs, tant sur la nécessité de modifier leur statut de "détenteur", pour celui de "commerçant " avec les restructurations que cela implique, que sur la nécessité d'une "politique de production ", avec maîtrise des approvisionnements et prévisions de fabrication.

Les nouvelles Commissions de la Forêt et des Produits forestiers continueront sans doute cette réflexion en commun et l'exercice de cette coresponsabilité dans la bonne marche de la filière, mais l'avance acquise en la matière à cause de cette catastrophe permet aujourd'hui d'accueillir des candidats à la transformation des bois d'un massif qu'on disait pourtant il y a quatre ans, irrémédiablement handicapé...

\section{L'ÉTAT PHYTOSANITAIRE DES FORÊTS}

Quel est, enfin, l'état sanitaire de la forêt du Massif Central après quatre campagnes de sensibilisation, de démonstrations, de surveillance, d'études et d'interventions auprès de tous les intéressés - ou supposés tels -...?

Les actions phytosanitaires sur les (hélas) futurs chablis seront-elles plus efficaces que dans le Massif Central où force est bien de constater, quand on traverse les pessières du Meygal (Haute-Loire) et qu'on longe maints peuplements des Combrailles ou du plateau de Millevaches, abandonnés à leur sort, sans exploitation, sans reconstitution, a fortiori sans le moindre piège à scolytes, que le Bostryche n'est pas éradiqué?

Pouvait-il en être autrement dans ces régions où la dispersion des trouées n'a d'égal que le morcellement des propriétés, rendant illusoire ou d'un coût exorbitant toute velléité de lutte organisée?

Mais si le Bostryche n'est pas encore vaincu, la lutte active menée depuis 4 ans par les forestiers, publics et privés, les agriculteurs (en Combrailles par exemple), les scientifiques ("Bio-Assistance" en Limousin), a créé un état d'esprit et un sens de l'observation précieux aujourd'hui tant pour détecter de nouveaux parasites que pour évaluer les diverses atteintes aux peuplements (réseau DEFORPA par exemple).

L'apparition de nouveaux parasites forestiers, en relation primaire avec la tornade (Hylésine du Pin) ou plus secondaire (Dendroctone, en phase d'extension) ou plutôt liée aux extrêmes climatiques récents (Tordeuse du Sapin de Haute-Loire, Pissode) montre l'intérêt d'une démultiplication des actions possibles par des organismes de défense à ce niveau cantonal, qui n'avait malheureusement pas été envisageable il y a 4 ans pour lutter contre les Bostryches.

Cette cémultiplication reste d'actualité car non seulement la lutte contre le Bostryche est loin d'être terminée, mais nous ignorons l'extension exacte des autres parasites précités.

\section{CONCLUSIONS}

Pour ne pas laisser les lecteurs sur ces questions pessimistes ou sans réponse (mais nos collègues allemands, avec beaucoup d'Épicéas, ont eu pendant 10 ans des difficultés avec leurs Bostryches) et sans reprendre les conclusions partielles tirées des différents volets de l'action menée pour effacer les dégâts de la tornade de 1982, on peut rappeler les quelques résultats les plus encourageants: 
- grâce à la conjonction des efforts et aux réunions fréquentes entre ses responsables publics (État et régions) et socio-professionnels, la "filière-bois " s'est relativement bien définie et s'organise peu à peu dans le Massif Central ; tant en Auvergne où le tissu des industries du bois, déjà dense, se rapproche de l'aval, qu'en Limousin où les producteurs et les industriels ont su prendre la mesure des problèmes d'écoulement du bois auxquels ils sont actuellement confrontés de par la montée en production de leur massif;

- des besoins en formation professionnelle se sont fait jour et des cycles ont été mis en place dès 1985 : formations à l'éclaircie linéaire, à l'utilisation d'engins forestiers, à la conduite de scieries modernes et à la qualité du sciage (affûteurs), surtout en Auvergne car le Limousin, avec l'Ecole de Meymac et l'Association pour le Développement des Éclaircies (ADELI) avait une certaine avance dans ces domaines;

- quatre ans de réflexion commune de l'ensemble des partenaires, pour les chablis d'abord, la charte ensuite, les futures ORF enfin, ont sorti la forêt de ses " hauts plateaux " ou de ses "fonds de vallées": elle est mieux perçue comme "productrice par les protecteurs et protectrice par les producteurs".

L'argumentation entre partenaires de la filière a d'ores et déjà dépassé les lieux communs de jadis et les actions nouvelles, étudiées de concert, rencontrent moins de blocages.

Cette réflexion en commun des scieurs, des experts et de l'Administration sur les problèmes de développement des entreprises, sur les choix - plus techniques - de matériels et d'organisation ou sur les difficultés financières et commerciales de la filière, a d'ailleurs permis de présenter à l'ensemble des responsables réunis au sein des Conseils régionaux de la Forêt et des Produits forestiers (en Auvergne notamment) des diagnostics et même des propositions d'actions coordonnées prioritaires.

Si le bond en avant, que la filière-bois du Massif Central est en train d'effectuer grâce aux efforts, au dévouement et à l'intelligence de tous ses partenaires, peut grâce aux actions proposées, la mettre plus à même de relever le défi des 1,5 million de $\mathrm{m}^{3}$ annuels supplémentaires, maintenant très proche, le prix payé en 1982 par les propriétaires forestiers, et les années suivantes par les scieurs, ne l'aura pas èté en vain... filière-bois ne permettait pas des adaptations à ses spécificités locales : morcellement de la propriété forestière, faible taille et fragilité financière et commerciale des entreprises.

$\mathrm{Si}$ ces handicaps expliquent des degrés divers dans la réussite des actions, tant en amont (lutte contre les bostryches, reconstitution des peuplements), qu'en aval (difficultés actuelles de certaines entreprises très endettées), la nécessité de les minimiser, sinon de les surmonter, a eu de nombreuses conséquences positives. 


\section{T. THURET}

Outre la sensibilisation réussie de nombreux forestiers aux problèmes techniques (parasites, choix d'essences et éclaircies) ainsi qu'à la formation et la sécurité, il faut surtout mentionner d'énormes progrès en matière d'insertion des différents partenaires dans l'organisation interprofessionnelle, la manifestation tangible de l'intérêt des élus locaux pour le secteur de la forêt et du bois et la montée en puissance et en qualité des industries locales qui pourront, en grande partie, répondre elles-mêmes au défi de l'accroissement attendu de production des forèts du Massif Central.

\section{WHAT IS THE SITUATION FOUR YEARS AFTER THE TORNADO OF 1982 IN THE MASSIF CENTRAL ? (SUmmaIY)}

Because of the urgent nature of the measures to be taken and the public assistance to be set up to minimise the consequences for the wood industry of the tornado of 1982, it was not possible to adapt them to specitic local factors: the division of the forest land, and the small size and financial and commercial instability of the enterprises.

While these disadvantages may explain the varying degrees of success of the measures, whether good (contral of bostrychio beetles, reconstruction of stands) or bad (present difficulties of some enterprises which are heavely in debt), the need to minimise them, if not to overcome them, has had many positive effects.

In addition to successfully making many foresters aware of the technical problems (parasites, choice of species and thinnings) as well as training and safety, mention must also be made of the huge progress in the integration of various partners into the interprofessional organisation, the tangible expression of interest in the forest and wood industries by local representatives, and the increase in the power and quality of local industries which could to a large extent react themselves to the challenge of the expected increased production of the forests of the Massif Central.

WIE SIEHT ES VIER JAHRE NACH OEM WIRBELSTURM VON 1982 IM ZENTRALMASSIV AUS? (Zusammenfassung)

Die Dringlichkeit der Massnahmen und der öffentlichen Unterstützungen, die es einzusetzen galt, um die Auswirkungen des Wirbelsturmes von 1982 auf die Holzwirtschaft zu mässigen, erlaubten es nicht sie den lokalen Gegebenheiten anzupassen : Parzellierung des Waldbesitzes, geringe Grösse sowie finanzielle und kaufmännische Schwäche der Unternehmen.

Wenn diese Nachteile die unterschiedlichen Abstufungen des Gelingens der Massnahmen erklären, sowohl was die Vorbeugung (Bekämpfung des Bostrich, Wiederaufforstung) als auch die Folgen (gegenwärtige Schwierigkeiten einiger stark verschuldeter Unternehmen) angeht, so hat doch die Notwendigkeit, sie zu verringern oder zu bekämpfen, zahlreiche positive Folgen gehabt. Abgesehen davon, dass das interesse vieler Forsleute sowohl für die technischen Probleme (Insektenbefall, Wahl der Arten und der Hiebe) als auch für die Fortbildung und die Sicherheit geweckt wurde, muss man vor allem die grossen Fortschritte auf dem Gebiet der Eingliederung der verschiedenen Partner in eine interprofessionelle Organisation, das Interesse der Lokalpolitiker für den Forst- und Holzzweig und den quantitativen und qualitativen Aufstieg der örtlichen Industriezweige erwähnen, die grösstenteils selbst dem voraussehbaren Anwachsen der Holzproduktion der Wälder des Zentralmassivs werden Rechnung tragen können. 